\title{
Nuclear modification of heavy quark fragmentation function and $J / \psi$ production in ultrarelativistic heavy ion collisions
}

\author{
Bin Zhang, Bao-An Li, Andrew T. Sustich, and Charles Teal \\ Department of Chemistry and Physics, Arkansas State University, \\ P.O. Box 419, State University, Arkansas 72467-0419, USA
}

(May 22, 2002)

\begin{abstract}
In ultrarelativistic heavy ion collisions, charm quark fragmentation is modified due to the melting of strings inside the high density partonic matter. $D$ mesons produced in hadronization can further produce $J / \psi$ particles. Using a multiphase transport model, we investigate the effect on the rapidity distribution of the produced $J / \psi$ particles with two different charm quark fragmentation functions. It is shown that the $J / \psi$ rapidity distribution is sensitive to the nuclear modification of charm quark fragmentation and thus is a good indicator of the onset of string melting and the production of deconfined partonic matter.
\end{abstract}

25.75.Dw,24.10.Lx,24.10.Jv

One interesting topic in strong interaction physics that has attracted much attention is the study of the phase transition between hot and dense hadronic matter and the Quark-Gluon Plasma (QGP) [1]. The QGP is believed to have existed in the early universe. It may also exist in the cores of neutron stars. Knowledge about the QGP phase transition is critical to the understanding of strong interaction physics and several other processes in cosmology and astrophysics. Several accelerators have been built and many experimental observables have been proposed for the search of the QGP [2,3]. $J / \psi$ production is one of the most promising probes for the formation of the QGP [1 22]. In this Letter, we demonstrate that due to the melting of strings inside a QGP, the heavy quark fragmentation function is modified. This modification is reflected clearly in the $J / \psi$ rapidity distribution. The measurement of the latter thus can be used to study the onset of string melting and the production of the QGP.

In relativistic nuclear collisions, the charm quark distributions can be calculated from perturbative quantum chromodynamics (pQCD). After the parton evolution, these charm quarks are converted into $D$ mesons. The $D$ meson distributions are determined by the charm quark distributions and the fragmentation function. In hadron induced reactions, charm quark fragmentation can be described by the delta function fragmentation function [23],

$$
D_{c}^{D}(z)=\delta(z-1) .
$$

Charm quark fragmentation in $e^{+} e^{-}$annihilation can be well described by the Peterson fragmentation function [24],

$$
D_{c}^{D}(z)=\frac{N}{z\left(1-1 / z-\epsilon_{c} /(1-z)\right)^{2}} .
$$

In the above equations, $z$ is the fractional momentum of the $D$ meson relative to the charm quark. $N$ is a normalization factor, and $\epsilon_{c} \sim\left(m_{q} / m_{c}\right)^{2}$. An explanation of the observed harder $D$ meson distribution in hadron induced interactions is provided by the Lund fragmentation model. In the Lund model [25] based event generator, PYTHIA [26], the charm quark is always at the end of a string and can be pulled by the faster valence particles to acquire a harder distribution.

In nuclear collisions, two scenarios can happen. In scenario 1, the produced charm $(c)$ and anticharm $(\bar{c})$ quarks are pulled by the strings of the receding nuclei. These strings break up after being stretched. A $c$ quark then combines with a light antiquark $(\bar{q})$ to form a $D$ meson. A similar process happens for the formation of the $\bar{D}$ meson (Figure 1). In scenario 2, the $c$ and $\bar{c}$ quarks are produced together with many gluons $(g)$. The interactions between the charm quarks and the receding nuclei are screened, and the strings between them melt [27,28]. At a later time, gluons fragment into quark-antiquark pairs, while other quark-antiquark pairs are also produced by the wounded nucleons. A $D$ meson can be formed by the combination of a charm quark and a light antiquark (Figure 2).

These two scenarios can lead to very different rapidity distributions for the produced $D$ and $D$ mesons. In scenario 1 , the charm quarks are pulled by the faster receding hadrons. The rapidity distribution will be harder and wider compared with that of scenario 2 in which the strings that pull the charm quarks are absent. In the second scenario, the charm quarks are also slowed down by other quarks, and the rapidity distribution will be softer and narrower. Charm quark fragmentation in scenario 1 can be modeled by the delta function fragmentation while that in scenario 2 can be modeled by the Peterson fragmentation function.

In ultrarelativistic nuclear collisions, the initial stage is dominated by minijet gluons. In particular, for $\mathrm{Au}+\mathrm{Au}$ collisions at $200 \mathrm{~A} \mathrm{GeV}$, the pQCD calculated gluon rapidity density is about 300 at midrapidity [29]. For comparison, 
the charm and anticharm rapidity density is about 3 [29]. In other words, these charm and anticharm quarks are immersed in a "sea" of hot gluons. Unlike for nuclear collisions at lower energies, or hadron-hadron collisions, the large amount of gluons modify the interactions between the charm quarks and the valence quarks. The strings between them melt when the gluon density is high. Several calculations have demonstrated the possibility of string melting at the Relativistic Heavy Ion Collider (RHIC) energies [27,28]. The melting of strings then leads to the absence of the pulling force that is responsible for the harder $D$ meson distribution observed in hadron induced reactions. This modification of the fragmentation function of charm quarks therefore reflects the production mechanism of the parton matter in ultrarelativistic nuclear collisions.

We propose to use the $J / \psi$ rapidity distribution as a measure of this modification. $J / \psi$ production is affected by several factors. Due to color screening inside the QGP, $J / \psi$ particles are dissociated above a certain temperature. A $J / \psi$ can be produced by the collision of a $c$ quark and a $\bar{c}$ quark when the temperature is below the dissociation temperature. In the parton phase, a $J / \psi$ can be destroyed by its collision with a minijet gluon. Similar processes can occur in the hadron phase. A $J / \psi$ can be produced by the collision of a $D$ meson and a $\bar{D}$ meson. It can be dissociated by the collision with a meson that is made of light quarks. The above $J / \psi$ production from final state hadronic interactions relates the $J / \psi$ rapidity distribution closely to the $D$ and $\bar{D}$ meson distributions.

The modification of charm quark fragmentation and its reflection in the $J / \psi$ rapidity distribution are studied by using a multiphase transport model [30 32]. This model is a hybrid model that is made up of several Monte Carlo models. The heavy ion jet interaction generator (HIJING) [33,34] is used to simulate the passing of two heavy nuclei and the production of the initial parton system. Zhang's parton cascade (ZPC) 35] is then used to study the evolution of this parton system. The conversion of the parton matter into hadron matter when no more parton interactions are available is done using the Lund fragmentation [25] that is part of the PYTHIA/JETSET Monte Carlo package [26]. A relativistic transport (ART) [36, 37] model will follow the hadron evolution until freeze-out. The multiphase model incorporates the major channels in different stages. It has been used to study particle production at RHIC energies and can successfully describe the global feature of the newly available RHIC data.

For $J / \psi$ production, $c+\bar{c} \leftrightarrow J / \psi+g$ is included in the parton stage and $D+\bar{D} \leftrightarrow J / \psi+M$ is included in the hadron stage. Here $M$ is a meson that is composed of one light quark $(q)$ and one light antiquark $(\bar{q})$. In relativistic nuclear collisions, charm quarks are rare particles. To effectively simulate the evolution of charm particles and $J / \psi$ 's, the perturbative method [38,39] is used. In this approach, charm quarks from several independent events are allowed to evolve with a common hadron environment. Since charm quarks are rare particles, their effects on the common hadronic environment are neglected.

We will compare the $J / \psi$ rapidity distributions in relativistic nuclear collisions for the two fragmentation scenarios. The multiphase transport model is used to simulate central $\mathrm{Au}+\mathrm{Au}$ collisions at RHIC at a center of mass energy, $\sqrt{s}=200 \mathrm{~A} \mathrm{GeV}$. The PYTHIA model is used to generate the initial charm and anticharm quarks. The charm production cross section per nucleon-nucleon collision is chosen to be $350 \mu \mathrm{b}$. The mass of the charm quark is chosen to be $1.50 \mathrm{GeV}$, and the mass of the $D$ mesons is set to be $2.01 \mathrm{GeV}$. A $3 \mathrm{mb}$ cross section is used for the elastic collisions between gluons and charm quarks and for the $J / \psi$ dissociation by gluons and light mesons. The cross sections of the inverse processes are calculated by using the detailed balance relation. These cross sections are consistent with previous PQCD and hadron model calculations [23,40 45].

The rapidity distributions of the $J / \psi$ particle, together with those for the charm quarks, $D$ and $\bar{D}$ mesons are calculated. Figure 3 shows the results for the case in which the delta function fragmentation is used to turn the charm quarks into $D$ mesons. It is seen that initial charm quark rapidity distribution has a plateau with a value of about 3.6 around $y=0$. After the parton evolution, the final charm quark rapidity distribution is almost the same as the initial distribution. This distribution leads to a $J / \psi$ rapidity distribution that also has a plateau with a value of about $0.7 / 300$ around $y=0$. The rapidity distribution for the $D$ and $\bar{D}$ mesons produced from hadronization is similar to the charm and anticharm quark distribution. The value around $y=0$ is about 4.3 and remains almost unchanged during the hadron evolution. $J / \psi$ particles are produced further in the hadron stage and the final rapidity distribution follows that of the $D$ and $\bar{D}$ mesons. The final value of $J / \psi$ particle around $y=0$ is about $1.9 / 300$.

The distributions for the Peterson fragmentation case are shown in Figure 1 . The initial, final charm quark distributions and the $J / \psi$ distribution after the parton stage are the same as those for the previous case since the parton stage has no information about the hadronization. As expected, the $D$ and $\bar{D}$ rapidity distribution becomes narrower by about 0.6 units of rapidity and has a high peak with a value about 6.4 at $y=0$. Notice that the total number of charm quarks does not change during hadronization. Hence, the area under the rapidity distribution of the charm and anticharm quarks equals that of the $D$ and $\bar{D}$ mesons. However, the production of $J / \psi$ particles is now starting from a $D$ and $\bar{D}$ rapidity distribution that is different from that of the delta function fragmentation case. This leads to a final $J / \psi$ distribution that is also narrower and has a peak in the middle with a value of about $5.6 / 300$ around $y=0$. 
In conclusion, the production of deconfined matter modifies the fragmentation of the charm and anticharm quarks. In nuclear collisions at lower energies, the string dynamics is important, and the charm fragmentation is similar to that in the hadron collisions. In nuclear collisions at ultrarelativistic energies, the minijet dynamics becomes important, and the charm fragmentation is more like the independent fragmentation in electron-positron collisions. This modification leads to a rapidity distribution of $J / \psi$ distinctively different from what is normally expected in hadronic reactions. The $J / \psi$ rapidity distribution measurement, in conjunction with information about charmed mesons, can offer valuable insight about the melting of strings and the production of the QGP in relativistic nuclear collisions at RHIC.

This work was supported in part by the Arkansas Science and Technology Authority under Grant No. 01-B-20, and by the U.S. National Science Foundation under Grant No. 0088934. We thank Z.W. Lin for helpful discussions. We also thank the Parallel Distributed System Facility at the National Energy Research Scientific Computer Center for providing computer resources. B.A. Li would like to thank S. Das Gupta and C. Gale for their kind hospitality at McGill University where part of this work was done.

[1] F. Wilczek, Phys. Today 53, 22 (2000).

[2] B. Müller, Rep. Prog. Phys. 58, 611 (1995).

[3] M. Gyulassy, Prog. Theor. Phys. Suppl. 140, 68 (2000).

[4] T. Matsui and H. Satz, Phys. Lett. B 178, 416 (1986).

[5] E.V. Shuryak, Sov. J. Nucl. Phys. 28, 408 (1979).

[6] X.M. Xu, D. Kharzeev, H. Satz, and X.N. Wang, Phys. Rev. C 53, 3051 (1996).

[7] S. Gavin and R. Vogt, Phys. Rev. Lett. 78, 1006 (1997).

[8] D. Kharzeev, C. Lounrenco, M. Nardi, and H. Satz, Zeit. Phys. C 74, 307 (1997).

[9] A. Capella, E.G. Ferreiro, and A.B. Kaidalov, Phys. Rev. Lett. 85, 2080 (2000).

[10] C. Spieles, R. Vogt, L. Gerland, S.A. Bass, M. Bleicher, L. Frankfurt, M. Strikman, H. Stöcker, and W. Greiner, Phys. Lett. B 458, 137 (1999). C. Spieles, R. Vogt, L. Gerland, S.A. Bass, M. Bleicher, H. Stöcker, and W. Greiner, Phys. Rev. C 60, 054901 (1999).

[11] W. Cassing and C.M. Ko, Phys. Lett. B 396, 39 (1997). J. Geiss, C. Greiner, E.L. Bratkovskaya, W. Cassing, and U. Mosel, Phys. Lett. B 447, 31 (1999).

[12] D.E. Kahana, S.H. Kahana, Phys. Rev. C 59, 1651 (1999).

[13] B.H. Sa, A. Tai, H. Wang, and F.H. Liu, Phys. Rev. C 59, 2728 (1999).

[14] C.M. Ko, X.N. Wang, B. Zhang, and X.F. Zhang, Phys. Lett. B 444, 237 (1998). P. Braun-Munzinger and Krzysztof Redlich, Eur. Phys. J. C 16, 519 (2000).

[15] R. Vogt, Nucl. Phys. A661, 250c (1999).

[16] W. Cassing, E.L. Bratkovskaya, S. Juchem, Nucl. Phys. A674, 249 (2000).

[17] B. Zhang, C.M. Ko, B.A. Li, Z. Lin, and B.H. Sa, Phys. Rev. C 62, 054905 (2000).

[18] C. Gale, S. Jeon, and J. Kapusta, Phys. Rev. C 63, 024901 (2001).

[19] R.L. Thews, M. Schroedter, and J. Rafelski, Phys. Rev. C 63, 054905 (2001).

[20] P. Braun-Munzinger and J. Stachel, Phys. Lett. B 490, 196 (2000). P. Braun-Munzinger, Nucl. Phys. A690, 119 (2001).

[21] L. Grandchamp and R. Rapp, Phys. Lett B523, 60 (2001).

[22] B. Zhang, C.M. Ko, B.A. Li, Z.W. Lin, and S. Pal, Phys. Rev. C 65, 054909 (2002).

[23] R.V. Gavai, S. Gupta, P.L. McGaughey, E. Quack, R.V. Ruuskanen, R. Vogt, and X.N. Wang, Int. J. Mod. Phys. A10, 2999 (1995).

[24] C. Peterson, D. Schlatter, I. Schmitt, and P.M. Zerwas, Phys. Rev. D 27, 105 (1983).

[25] B. Andersson, G. Gustafson, G. Ingelman, and T. Sjöstrand, Phys. Rep. 97, 31 (1983).

[26] T. Sjöstrand, Comput. Phys. Commun. 82, 74 (1994).

[27] Tamás S. Biró, B. Müller, and X.N. Wang, Phys. Lett. B283, 171 (1992).

[28] Z.W. Lin and C.M. Ko, Phys. Rev. C 65, 034904 (2002).

[29] X.N. Wang, Phys. Rep. 280, 287 (1997).

[30] B. Zhang, C.M. Ko, B.A. Li, and Z. Lin, Phys. Rev. C 61, 067901 (2000).

[31] Z.W. Lin, S. Pal, C.M. Ko, B.A. Li, and B. Zhang, Phys. Rev. C 64, 011902 (2001).

[32] Z.W. Lin, S. Pal, C.M. Ko, B.A. Li, and B. Zhang, Nucl. Phys. A698, 375c (2002).

[33] X.N. Wang and M. Gyulassy, Phys. Rev. D 44, 3501 (1991).

[34] M. Gyulassy and X.N. Wang, Comput. Phys. Commun. 83, 307 (1994).

[35] B. Zhang, Comput. Phys. Commun. 109, 193 (1998). 
[36] B.A. Li and C.M. Ko, Phys. Rev. C 52, 2037 (1995).

[37] B.A. Li, A.T. Sustich, B. Zhang, and C.M. Ko, Int. J. of Mod. Phys. 10, 267 (2001).

[38] J. Randrup and C.M. Ko, Nucl. Phys. A343, 519 (1980).

[39] X.S. Fang, C.M. Ko, and Y.M. Zheng, Nucl. Phys. A556, 499 (1993).

[40] B.L. Combridge, Nucl. Phys. B151, 429 (1979).

[41] M.E. Peskin, Nucl. Phys. B156, 365 (1979). G. Bhanot and M.E. Peskin, Nucl. Phys. B156, 391 (1979).

[42] K.L. Haglin, Phys. Rev. C 61, 031902 (2000).

[43] Z. Lin and C.M. Ko, Phys. Rev. C 62, 034903 (2000); W. Liu, C. M. Ko, and Z.W. Lin, ibid. 65015203 (2002).

[44] A. Sibirtsev, K. Tsushima, and A.W. Thomas, Phys. Rev. C 63, 044906 (2001).

[45] C.Y. Wong, E.S. Swanson, and T. Barnes, Phys. Rev. C 62045201 (2000); 65014903 (2002); C.Y. Wong, T. Barnes, E.S. Swanson, and H.W. Crater, e-Print archive: nucl-th/0112023. 
(a)

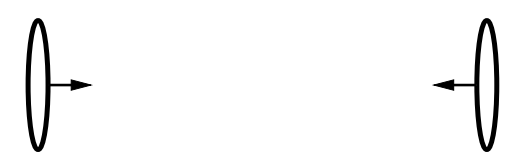

(b)

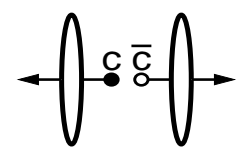

(c)

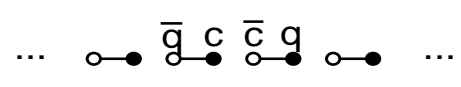

(d) $\ldots \bigodot \underbrace{\bar{g} g}_{D} \underbrace{\bar{z}}_{\bar{D}} \odot \ldots$

FIG. 1. Time evolution of the collision in fragmentation scenario 1. (a) Two heavy nuclei at relativistic speeds approach each other. (b) After the collision, a charm quark and an anticharm quark are produced. They are attached to the receding nuclei by strings. (c) At a later time, quark-antiquark pairs are produced from the strings. (d) The charm quark and a light antiquark combine to form a $D$ meson, while the anticharm quark and a light quark combine to form a $\bar{D}$ meson.

(a)
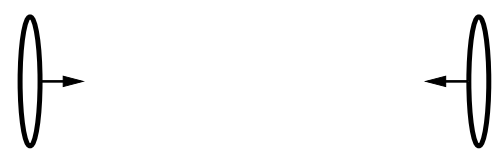

(b)

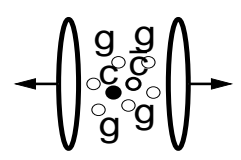

(c)

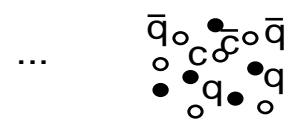

(d)

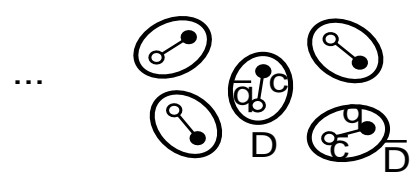

FIG. 2. Time evolution of the collision in fragmentation scenario 2. (a) Two relativistic nuclei approach each other. (b) A charm quark and an anticharm quark are produced together with a large number of gluons. (c) At a later time, light quark-antiquark pairs are produced. (d) The charm quark and a light antiquark combine to form a $D$ meson, while the anticharm quark and a light quark combine to form a $\bar{D}$ meson 


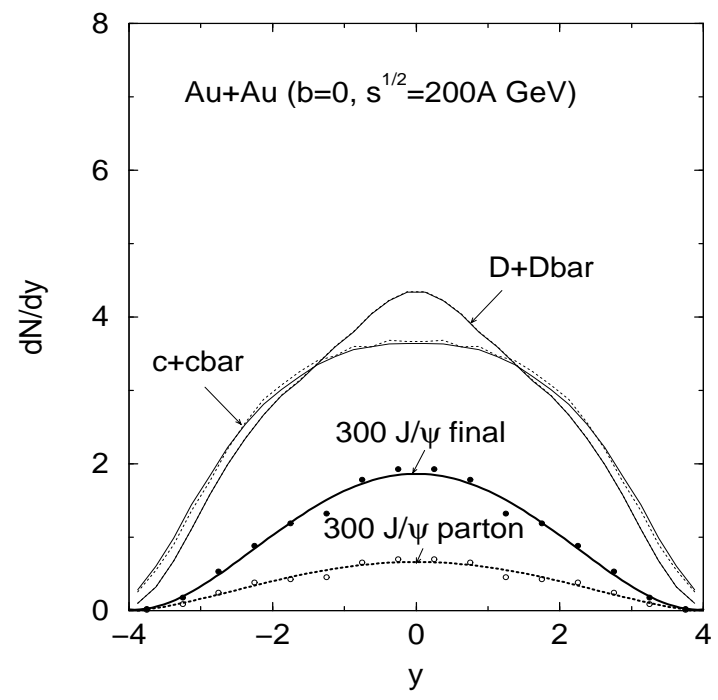

FIG. 3. Rapidity distributions of $J / \psi$ particles, charm and anticharm quarks, $D$ and $\bar{D}$ mesons for the delta function fragmentation case. The dotted line $J / \psi$ distribution is 300 times the distribution after the parton stage. The solid line $J / \psi$ distribution is 300 times the distribution after the hadron evolution. The dotted line charm and anticharm quark distribution is before the parton evolution and the solid line is after the parton evolution. The dotted line for the $D$ and $\bar{D}$ meson distribution is before the hadron evolution and the solid line is after the hadron evolution.

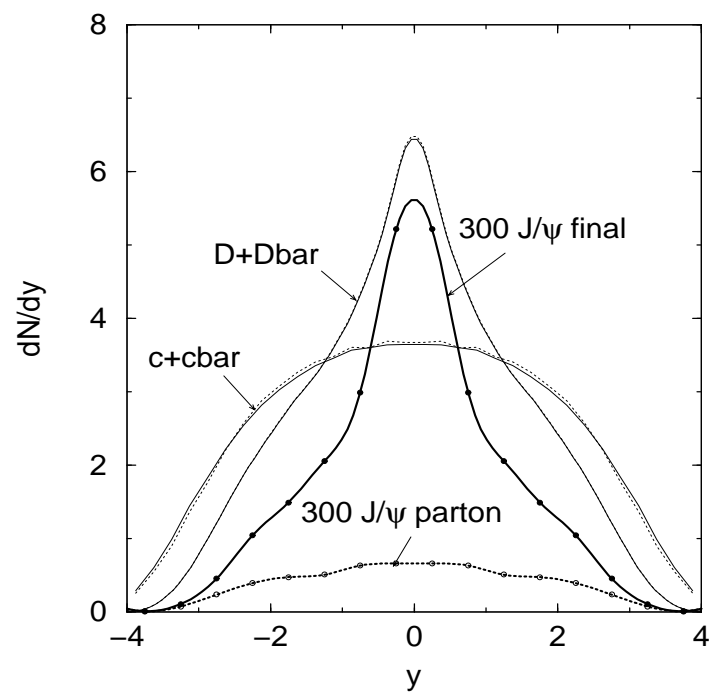

FIG. 4. Rapidity distributions of $J / \psi$ particles, charm and anticharm quarks, $D$ and $\bar{D}$ mesons for the Peterson fragmentation function case. The symbols are defined the same as those in Fig. 3 . 